\title{
FEDERICO RUBIO Y GALI, "PRÍNCIPE DE LA CIRUGÍA", Y LA UROLOGÍA EN LA SEVILLA DEL SIGLO XIX
}

\author{
Juan Manuel Poyato Galán, María del Mar García Millán' y María Felisa Álvarez Rey².
}

Unidad de Urología. Hospital de Alta Resolución de Utrera. Sevilla.

Oficina de Historia. Asociación Española de Urología.

"Servicio de Cirugía Plástica, Reparadora y Estética. Hospital Clínico Universitario "Virgen Macarena" de Sevilla.

${ }^{2}$ Departamento de Historia Contemporánea. Universidad de Sevilla. España.

Resumen.- El presente trabajo describe la figura del Dr. Federico Rubio y Galí, pionero e impulsor de la Medicina Española en el siglo XIX, así como la repercusión de su talento científico en el desarrollo de la Urología moderna. También se relaciona la creación de la Escuela Libre de Medicina y Cirugía de Sevilla, como puesta en práctica de su vocación docente y contribución a la enseñanza del conocimiento Urológico.

Palabras clave: Federico Rubio. Escuela Libre de Medicina. Historia de la Urología.
Summary.- The present work describes the figure of Dr.Federico Rubio y Gali, pioneer and promoter of Spanish Medicine during the 19th century, as well as the repercussion of his scientific talent in the development of modern urology. It is also mentioned the creation of the Free School of Medicine and Surgery of Seville, as concretion of his educational vocation and contribution to the teaching of urological knowledge.

Keywords: Federico Rubio. Free School of Medicine. History of Urology.

\section{INTRODUCCIÓN}

Sin lugar a dudas, se podría postular que el Doctor Don Federico Rubio y Galí es la personalidad más relevante y significativa de la Medicina española del siglo XIX. No obstante, tanto su figura como sus obras adolecen de un desconocimiento generalizado que, gracias a la reciente conmemoración del $175^{\circ}$ aniversario de su nacimiento (2002), ha logrado mitigarse en gran medida con la aparición de nuevos estudios que matizan la importancia de su figura.

El presente trabajo ilustra la relevancia científica de un destacado dinamizador de la enseñanza y desarrollo de la Medicina, piedra de toque a la vez de los inicios de la Urología en la Universidad española. Por añadidura, se ahonda en cómo la mentalidad liberal de su visión política encontró el caldo de cultivo idóneo en la sociedad sevillana decimonónica, como estímulo para el diseño sociopolítico de 
estructuras higiénicas y sanitarias que redundaran en una mejora de la salud de la población con menos recursos.

La época sevillana de Don Federico Rubio y Galí, por ser su primer ejercicio como figura docente con responsabilidad, puede que no sea de las más lucidas en el ámbito médico ni en el de las grandes intervenciones quirúrgicas, pero sí fructíferas en cuanto a lo que en producción de estudios e investigaciones médicas se refiere. Empero, es indiscutible que en la capital hispalense se sentaron las bases de importantes aportaciones para la incorporación de la Urología a la Cirugía Moderna, así como de su enseñanza como disciplina misma. Los años sevillanos de Don Federico Rubio y Galí constituyeron así mismo, el periodo más vital y filantrópico del joven doctor y también el de mayor participación política; por ello uno de los epígrafes de nuestro estudio está dedicado a ésta actividad.

Ya desde su juventud, y sustentado en parte por las diferencias de su ideología liberal demócrata con el orden político establecido, realizó visitas a los principales hospitales de Europa y más tarde

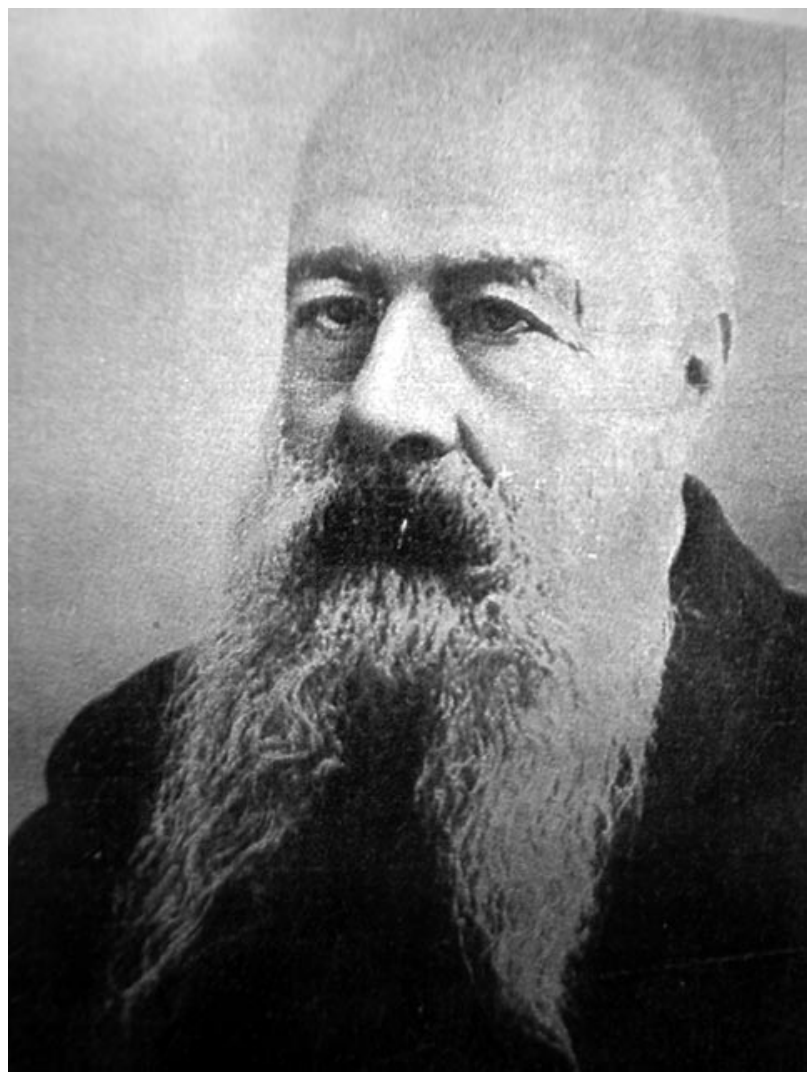

FIGURA 1. El Doctor Don Federico Rubio y Galí. de EE.UU. (estancias justificadas por diversos autores como periodos de exilio: 1859, 1873). Desde Sevilla marchó a Francia, y a su regreso intentó poner en práctica las innovaciones aprendidas, dado que Don Federico Rubio y Galí fue siempre un gran pedagogo cuyo sueño fue enseñar, transmitir los conocimientos aprendidos y formar profesionales de la Medicina con el mejor nivel académico y científico accesible en su época.

La importancia del Doctor Rubio y Galí queda atestiguada por la cantidad de proyectos realizados y títulos acumulados desde su juventud hasta los últimos años de su vida. Entre ellos, podemos contar los siguientes: La fundación en 1868 de la Escuela Libre de Medicina y Cirugía de Sevilla, pionera y modelo en su época. En 1873 fue nombrado Miembro de Honor del Royal College of Surgeons de Londres, concediéndosele el título de "Príncipe de la Cirugía". En el mismo año de 1873 fue designado Embajador en el Reino Unido por el gobierno de la I República Española, regresando a Madrid como Consejero de Estado. En 1874 fue nombrado miembro de la Academia Nacional de Medicina y en 1880 se encargó de la creación del Instituto de Terapéutica Operatoria en el Hospital de la Princesa de Madrid; el conocido como Instituto Rubio, origen de la Cirugía moderna en España. En 1895, preocupado por la formación de los profesionales sanitarios creó la Escuela de Enfermería de Santa Isabel de Hungría, siguiendo el modelo de la de Florence Nightingale, que conoció ampliamente durante su estancia en Londres.

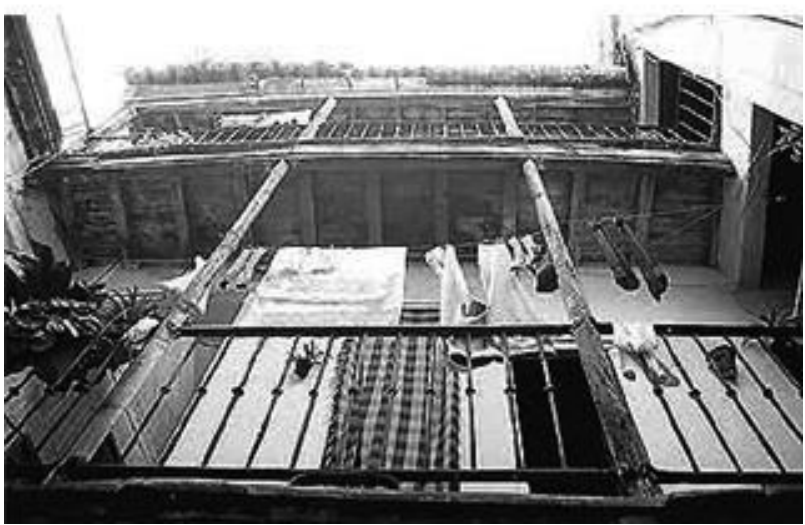

FIGURA 2. Corral de vecinos de la calle San Clemente, Sevilla. Típica vivienda de gente humilde en la que las habitaciones y la vida familiar giraban alrededor de un patio común. Siglo XIX. 
En suma, Don Federico Rubio y Galí fue médico prestigioso y político insigne, que se preocupó por los problemas de su tiempo y en todo momento mantuvo una actitud comprometida con la sociedad a lo largo de toda su vida. Se preocupó por elevar la Medicina, ya fuera en su ejercicio ya en su enseñanza, al mayor nivel de conocimiento posible en su época con una tenacidad e interés encomiables, siempre decidido a que la asistencia sanitaria llegara a los sectores más desprotegidos de la población. Desde el punto de vista de la Urología, se le considera el médico que más contribuyó a la creación e impulso de ésta especialidad, aún hoy día sin lograr escapar a la controversia que lógicamente suscitaba un profesional pionero, sobresaliente en el contexto de la desconfianza de unos colegas científicamente inmovilistas y reacios a los avances llegados de fuera. Pero también fue un personaje cuestionado y no exento de polémica debido a su pensamiento /o talento) político, indudablemente empleado por el Doctor Rubio y Galí como instrumento para trasladar a la realidad sus planes de repercusión higiénica y mejoras sociales.

\section{Los años sevillanos de Don Federico Rubio y Galí (1854-1873)}

No resulta fácil encontrar una figura que reúna los arquetipos propios de un personaje típico del siglo XIX sevillano como el Doctor Don Federico Rubio y Galí. Poco sabemos de su biografía y, aunque su nacimiento y formación se produjo en la localidad gaditana de El Puerto de Santa María, parte importante de su vida profesional se desarrolló en Sevilla.
Con veintitrés años Don Federico Rubio terminó sus estudios de Medicina en Cádiz, de la mano de maestros destacados como José de Gardoqui y Manuel José de Porto y no sin grandes esfuerzos económicos. Los años de estudio de Don Federico Rubio coinciden con la época isabelina (1834-1868), un período de grandes dificultades, crisis y guerras carlistas. Destacó entre sus compañeros desde el principio, sobre todo por sus amplios conocimientos anatómicos. Ésta y otras habilidades le proporcionaron el puesto de Ayudante de Director. Un año antes de acabar sus estudios, en 1849, publicó la que sería su primera obra de una larga lista de publicaciones: "Manual de Clínica Quirúrgica".

En el siglo XIX la capital andaluza era una ciudad decadente en la que nada quedaba del viejo esplendor de la "Sevilla Americana". La crisis económica había provocado la ruina de las pocas Academias existentes y hecho desaparecer la Facultad de Medicina; pese a su eclipse, Sevilla supo mantener el pulso y su eterno poder de atracción de gran urbe, particularmente entre las poblaciones de su entorno, capaz de ofrecer oportunidades para recién-licenciados. En el caso de la Medicina sevillana, esta oportunidad se materializaba en la oposición a una plaza de Cirujano del Hospital Central, lo que motivó al joven doctor gaditano a abandonar su ciudad natal en busca de una oportunidad profesional. Pese a sus indudables méritos y capacidades, Don Federico Rubio no consiguió la plaza a la que optaba, motivando una fuerte polémica que llegó a la prensa de la época causando gran tumulto; puede ser que sus ideales políticos le impidieran injustamente lograrla. Pese a este primer fracaso en la ciudad sevillana,

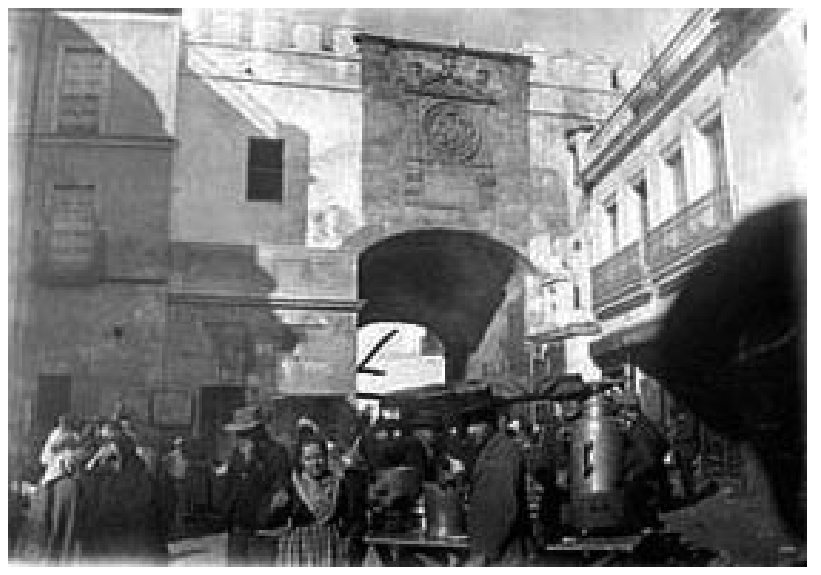

FIGURA 3. Mercado del aceite. Arco del Postigo, Sevilla. Siglo XIX.

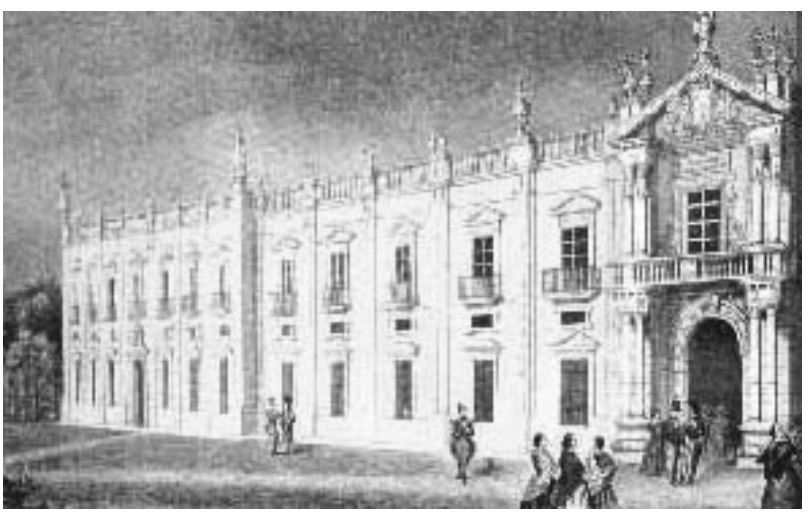

FIGURA 4. Fachada principal del edificio de la Universidad de Sevilla (Real Fábrica de Tabacos). Año 1505. 


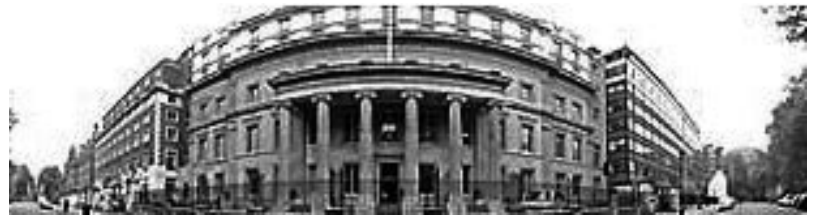

FIGURA 5. Edificio del Royal College of Surgeons, Londres, institución que concedió al Dr. Rubio y Gali el título de "Príncipe de la Cirugía".

Don Federico también encontró muestras de apoyo suficientes como para decidir quedarse en Sevilla y comenzar su ejercicio profesional.

El Doctor Rubio y Galí no dudó en utilizar su posición política para beneficiar en lo posible determinados aspectos relacionados con la educación y práctica médica. Uno de sus mayores logros en éste sentido lo constituyó la creación en 1868 de la Escuela Libre de Medicina y Cirugía en la capital hispalense, de la que más tarde nos ocuparemos.

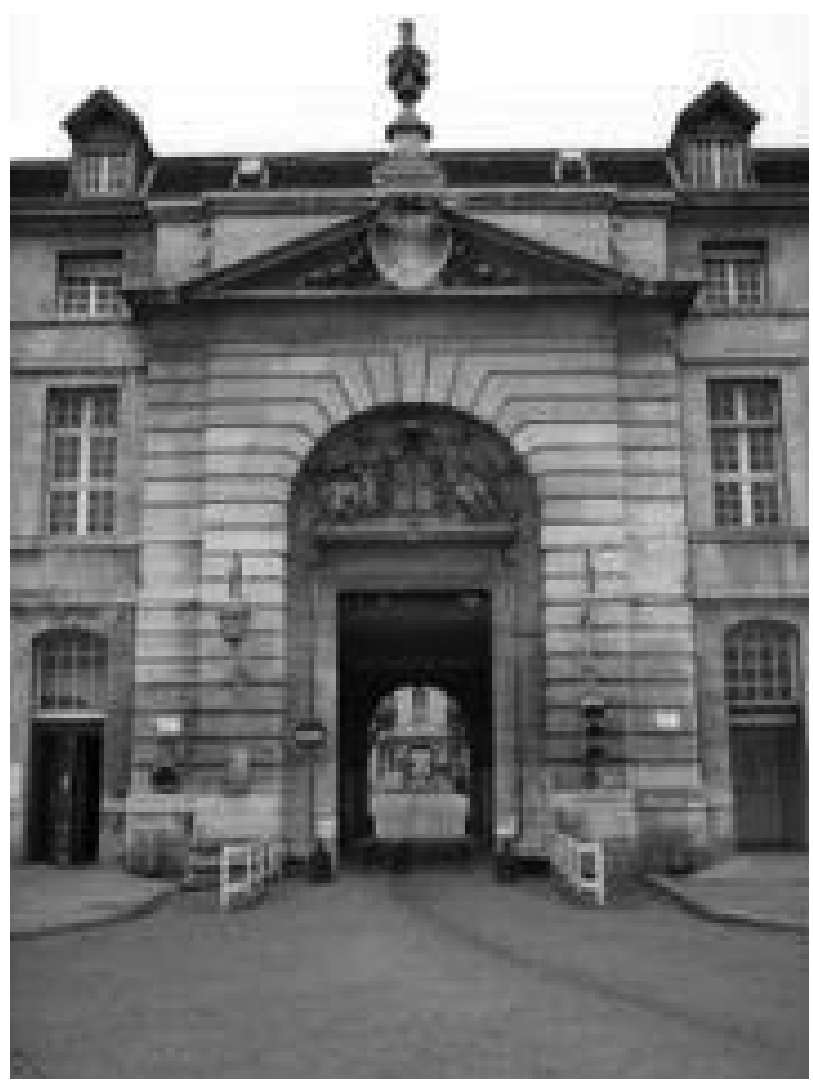

FIGURA 6. Hospital de La Pitiè, París, lugar de visita e intercambio académico para el Dr. Rubio y Galí.
En Sevilla, los ojos de Don Federico Rubio contemplaron el aspecto desolador y la miseria existente en las calles de una capital con unas infraestructuras muy deficitarias: el alcantarillado, cuando existía, era precario y con desagües insuficientes, lo cual favorecía la formación de grandes charcas que se convertían en fuente constante de infecciones.

No debemos olvidar que en el siglo XIX se sucedían enfermedades epidémicas como la fiebre amarilla, el cólera, la tuberculosis, la viruela, el sarampión, la escarlatina, la difteria o el tifus; enfermedades que se cebaban especialmente en los más menesterosos y que, sin vacunas existentes, provocaban elevadas tasas de mortalidad, especialmente entre la población infantil. Pero sin ninguna duda, una de las enfermedades más temidas en la época era la Sífilis, enfermedad infecciosa, contagiosa y transmisible también conocida como "Mal Francés" o "Mal de Venus". En la época, por su poder pandémico, mortífero y la falta de remedio existente, bien podía ser comparable con el SIDA de hoy en día. A ésta gran carga mortal de la Lúes, se añadía un componente social al correlacionarse con la prostitución, lo que la hacía aún más temible en una sociedad muy influenciada por la moral y preceptos religiosos. Ante la dificultad para controlar la propagación de la Sífilis y de las enfermedades venéreas, el Doctor Rubio y Galí insistió en la profilaxis y en la educación sobre higiene personal como principal remedio, ya que la incertidumbre sobre su tratamiento no se resolverá hasta bien entrado el siglo XX con el descubrimiento de la penicilina.

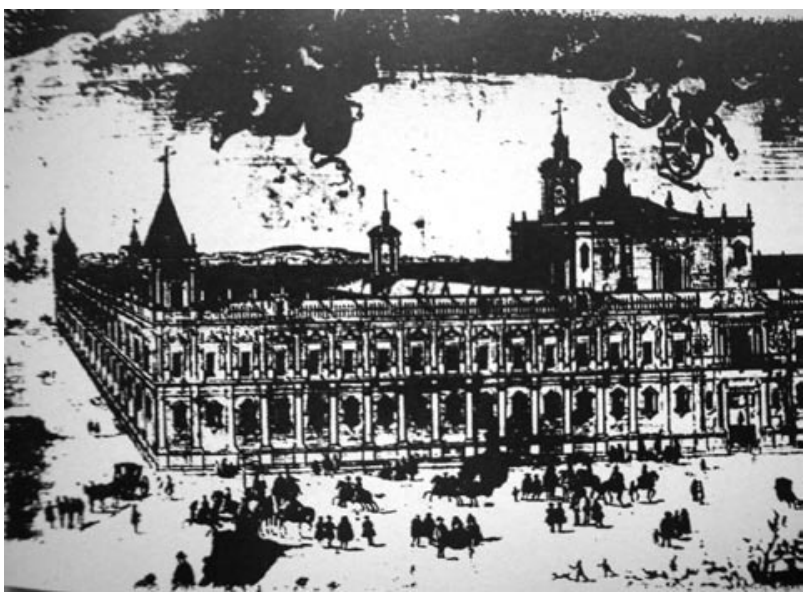

FIGURA 7. Hospital de la Sangre o De las Cinco Llagas, actual Parlamento de Andalucía. Imagen retrospectiva de 1559 
En el XIX se empezó a adoptar una nueva consideración hacia el enfermo, sustituyendo el sentido de caridad cristiana por otro, público, en el que correspondía al poder civil la responsabilidad de la asistencia sanitaria de forma gratuita. Pero muy lejano quedaba el concepto de universalidad asistencial, de tal manera que por entonces, las clases pudientes acudían a las consultas de médicos afamados, las clases medias eran visitadas en sus domicilios doctores de menor renombre, y los pobres se conformaban con ser admitidos en hospitales de beneficencia la todas luces deficientes tanto en su aspecto arquitectónico como en recursos humanos y materiales).

Ante ésta situación no es difícil imaginar a un joven Doctor Rubio y Galí abrumado y sobrepasado por la realidad.

En 1859 el Dr. Rubio y Galí fue detenido junto con otros demócratas y, exiliado, marchó a París donde tuvo la ocasión de aumentar sus conocimientos médicos. No es coincidencia que los centros de estudios parisinos elegidos: Hotel de Dieu, La Pitié, Saint Louis y Necker, tuvieran algo en común: El primero de ellos, Hotel de Dieu, el más antiguo de París y cerca de Notre Dame, representó el símbolo de la caridad y de la hospitalidad. La Pitié trataba a indigentes, niños abandonados y enfermedades incurables; Saint Louis gozaba de fama en el tratamiento de enfermedades contagiosas como la sífilis, y el Necker desde principios del siglo XIX se especializó en enfermedades infantiles. No son menos prestigiosos

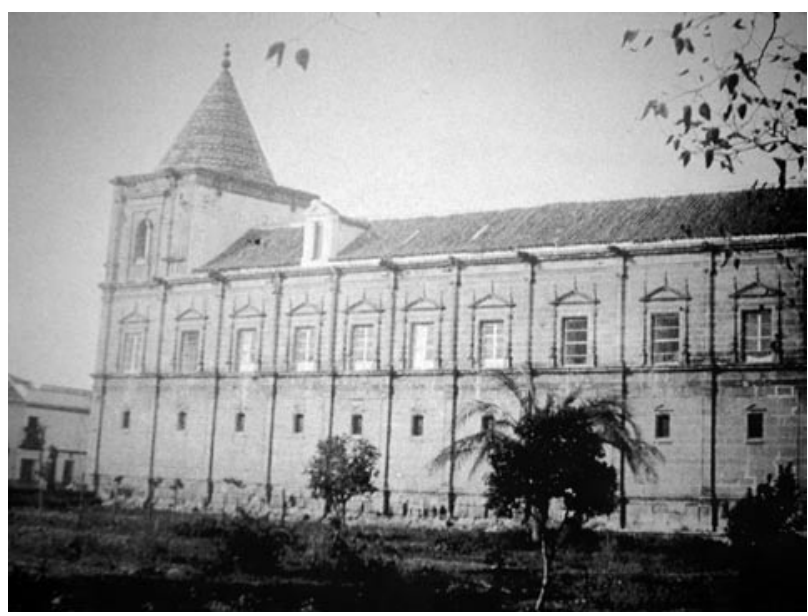

FIGURA 8. Vista parcial del Hospital de las Cinco Llagas a finales del Siglo XIX, cuando los alumnos de la Escuela Libre de Medicina y Cirugía recibían sus lecciones en sus dependencias. los doctores con los que trató Don Federico Rubio: Velpeau destacaba en anatomía quirúrgica, Nèlaton, profesor de clínica quirúrgica y uno de los primeros en introducir la ovariectomía en Francia, contribuyó a mejorar la técnica de cateterismo vesical e innovaciones instrumentales como la sonda exploratoria y el catéter que lleva su nombre. Con el Dr. Broca, profesor de la Universidad de Paris y notable investigador, el Doctor Rubio y Galí tuvo en común su preocupación por la salud de los más necesitados y la asistencia pública.

Don Federico Rubio y Galí regresó a Sevilla en 1865 donde intentó llevar a la práctica -así como durante toda su vida- los conocimientos adquiridos, luchando contra el paternalismo e insolidaridad de las clases dirigentes sin escatimar esfuerzos para conseguir el traspaso de una sanidad de carácter caritativo en manos de la Iglesia, a una sanidad pública que se correspondía con su concepto de justicia social. Defendió la importancia de la higiene, de la Medicina Preventiva individual y de la creación de hospitales y consultorios públicos. Supo percatarse de que había enfermedades que dependían directamente del nivel social y de los recursos familiares del individuo, donde la falta de higiene era crucial, correspondiéndose claramente con las teorías higienistas difundidas durante la época isabelina.

La gran oportunidad de poner en práctica sus ideales le llegó en 1868 con la creación en Sevilla de la Escuela Libre de Medicina y Cirugía, base de la futura Facultad de Medicina como se refirió anteriormente.

\section{La Escuela Libre de Medicina y Cirugía de Sevilla}

La enseñanza de la Medicina se impartió en Sevilla de manera ininterrumpida de 1572 a 1857 , fecha en la que desaparece la Facultad de Medicina en la ciudad hispalense, manteniéndose tan sólo la Facultad de Medicina de Cádiz. Del siglo XIX merece mención la creación durante el Trienio Constitucional (1820-1823) de la Universidad Libre de Medicina: Las Cortes liberarles aspiraban a lograr una transformación científica en profundidad, partiendo de una modernización de los estudios y de una mayor dotación de recursos materiales que hicieran posible su funcionamiento práctico. Si bien los continuos cambios políticos producidos durante el primer tercio del siglo XIX impidieron su aplicación, la enseñanza de la Medicina a lo largo de esta centuria no consiguió el ambiente propicio para su desarrollo, frenado por el férreo control e influencia de la Iglesia sobre la cultura y la educación. 
La Revolución de 1868 supuso el triunfo del liberalismo progresista. Las pretensiones de este movimiento, conocido como "La Gloriosa", desde el punto de vista educativo se centraron principalmente en los siguientes aspectos:

- Enseñanza libre

- Creación de nuevos centros educativos ante la desoladora situación de la enseñanza en Sevilla

- Autorización a todos los ciudadanos para fundar centros de enseñanza, independientes del control eclesiástico

- Introducción de cambios significativos en la enseñanza científica

- Libertad de cátedra en lo referido a doctrina y libros de textos

En este ambiente tan propicio para el desarrollo científico se creó la Escuela Libre de Medicina y Cirugía de Sevilla (en adelante Escuela) el 28 de octubre de 1868. Su sesión inaugural tuvo lugar en la Academia Sevillana de las Buenas Letras presidida por el Rector de la Universidad, Don Antonio Machado, abuelo de los poetas, y ostentando el cargo de director de la Escuela Don Antonio Marsella Sierra. Este último fue el encargado de pronunciar el discurso de apertura con la disertación "Sobre los problemas generales de la Ciencia".

La apertura de la Escuela representó la puesta en práctica de una mentalidad experimental que hasta entonces no había encontrado un espacio temporal adecuado para su desarrollo; ello vino a cubrir el vacío que en temas científicos pesaba sobre la ciu-

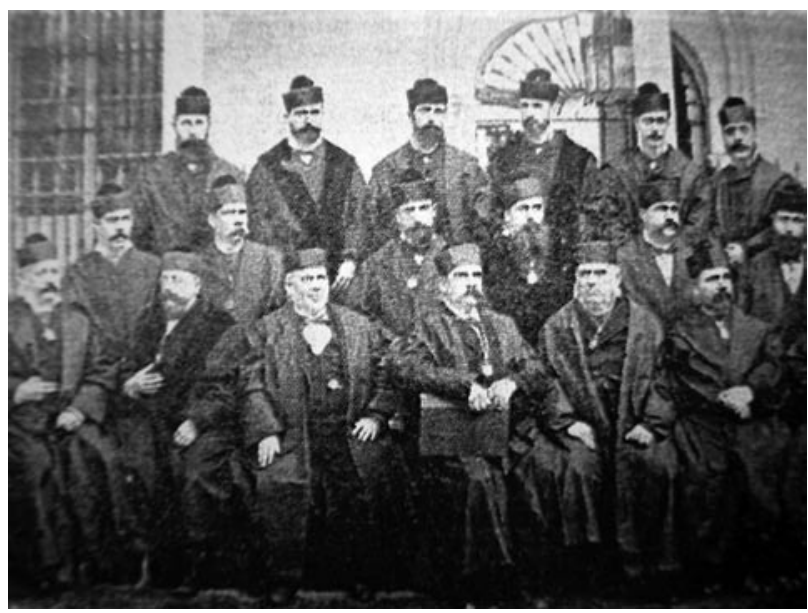

FIGURA 9. Claustro de profesores de la Escuela Libre de Medicina y Cirugía de Sevilla (1868). dad. Además, la Escuela suponía grandes esperanzas para una Sevilla en la que, desde la desaparición de la Facultad de Medicina en 1857, la notable decadencia de instituciones extrauniversitarias y el escaso nivel de la enseñanza médica hasta entonces, la atención sanitaria a la población resultaba a todas luces deficiente.

La implicación en el nacimiento de esta institución de la Corporación Municipal y de la Diputación Provincial de Sevilla fue inmediata en cuanto al apoyo económico y dotación de infraestructuras, siendo los propios poderes gubernamentales los que auspiciaron y protegieron a la Escuela desde su fundación. Aunque no se trató de un patrocinio desinteresado; el gobierno municipal, incapaz de enfrentarse día a día con una ciudad cada vez más poblada e insalubre, con una atención sanitaria insuficiente, mostró su interés por la implicación de la nueva institución en la paliación de los males de la ciudad. La protección se hizo tan evidente desde el primer momento de la fundación que la Diputación Provincial de Sevilla se convirtió en su principal bienhechor y poniendo a disposición de la Escuela el Hospital Central (Hospital de las Cinco Llagas, actual sede del Parlamento Andaluz). Con todos estos condicionantes se podría decir que el deseado carácter autónomo soñado por el Doctor Federico Rubio y Galí comenzó a enturbiarse hasta desaparecer poco tiempo después.

El plan docente de La Escuela Libre de Medicina y Cirugía de Sevilla contó con 18 asignaturas oficiales y varias complementarias, lo que la convirtió en un plan de estudios médicos muy completo para la España de la época. La creación de la Escuela y la novedosa metodología aplicada fueron consideradas como un logro personal del Doctor Federico Rubio, que detentó la Cátedra de Clínica Quirúrgica. No obstante, algunos autores se apoyan en la contratación de profesores de la antigua Facultad de Medicina de la década de los 40 (Ballesteros Sousa, Marsella Sierra y Rivera Ramos, así como a su propio maestro de Anatomía de Cádiz, Domínguez Daza), y en el empleo de planes de estudio diseñados previamente, para asentar un falso carácter revolucionario, aunque no comentan que nunca fueron desarrollados completamente por sus inspiradores (planes de Moyano y Orovio).

El 6 de noviembre de 1868 se abrió el periodo de matriculación que la Escuela exigía para su ingreso: ser bachiller en Artes y haber terminado los cursos de ampliación (Física experimental, Química y Zoología, Botánica y Mineralogía).

El plan docente de la Escuela presentaba novedades si no abismales, sí más que suficientes 
para distanciarse en algunos aspectos de la Enseñanza Oficial, destacando la importancia que desde un primer momento la Escuela concedió a la práctica médica. Esta preponderancia se materializó en la creación de una Policlínica con objetivos asistenciales de carácter gratuito y que atendió tanto consultas ambulatorias como a domicilio, con la participación de los profesores y alumnos de la Escuela.

Las clases dieron comienzo en diciembre de 1868 en las aulas de la Universidad hasta que en 1878 se trasladó al convento Madre de Dios, en el que permaneció hasta mediados del siglo XX cuando se trasladó al barrio de la Macarena.

Tabla I.
La enseñanza teórica se completaba con la práctica en la Policlínica. Los alumnos eran libres para matricularse en las asignaturas que desearan, si bien la Escuela agrupaba las asignaturas en cinco grupos: el primero y el segundo correspondientes a las de Anatomía y Fisiología, y los tres restantes a las Clínicas y Quirúrgicas. Lo habitual era finalizar los estudios en cinco años. Los cursos comenzaban el primero de septiembre y el primero de febrero, es decir de septiembre a enero y de febrero a junio.

El nivel del profesorado no era uniforme, ya que su elección estuvo limitada a la oferta existente, siendo genéricamente profesionales formados en Sevilla o Cádiz, sin experiencia docente previa, que ejercieran la Cirugía u otra especialidad Quirúrgica y con vocación de permanecer en la Escuela. El

\section{TABLA I. ASIGNATURAS IMPARTIDAS.}

\begin{tabular}{|l|l|}
\hline \multicolumn{1}{|c|}{ ASIGNATURA } & \multicolumn{1}{c|}{ PROFESOR } \\
\hline Anatomía Descriptiva ( 2 cursos) & Vicente Chiralt \\
\hline Anatomía General ( 1 curso) & Isidoro Díaz \\
\hline Ejercicio Osteología (30 lecciones) & Manuel Porrúa \\
\hline Ejercicio Disección (2 cursos) & Manuel Porrúa \\
\hline Fisiología (2 cursos) & Ramón Esteban Ferrando \\
\hline Higiene Privada (1 curso) & Jacinto Zaldo \\
\hline Patología Clínica General (1 curso) & Javier Pérez Lasso \\
\hline Anatomía Patológica ( 1 curso) & José Teodoro Muñoz de las Cajigas \\
\hline Patología Médica (2 cursos ) & José López del Baño \\
\hline Terapéutica, Materia Médica y arte de recetar (2 cursos ) & Antonio Marsella \\
\hline Clínica Médica (2 años solares) & Francisco Gómez \\
\hline Clínica Quirúrgica (2 años solares) & Federico Rubio Galí \\
\hline Clínica Obstétrica (1 año solar) & Joaquín Ballesteros \\
\hline Higiene Pública (1 curso) & Jacinto Zaldo \\
\hline Medicina Legal y Toxicología (1 curso) & Carlos Morón \\
\hline Historia, Filosofía y Moral Médica (1 curso) & Rafael Ariza \\
\hline Análisis Química Aplicada (1 curso ) & Isidro Vázquez \\
\hline Histología Normal y Patológica (1 curso) & Antonio Salado \\
\hline Oftalmología (1 curso) & Ramón de la Sota y Lastra \\
\hline Afectos Venéreos (1 curso) & Jerónimo Sánchez \\
\hline
\end{tabular}

Fuente: Trigueros Gordillo, G; "La Universidad de Sevilla durante el Sexenio Revolucionario". Universidad de Sevilla, Secretariado Publicaciones, Sevilla, 1898 


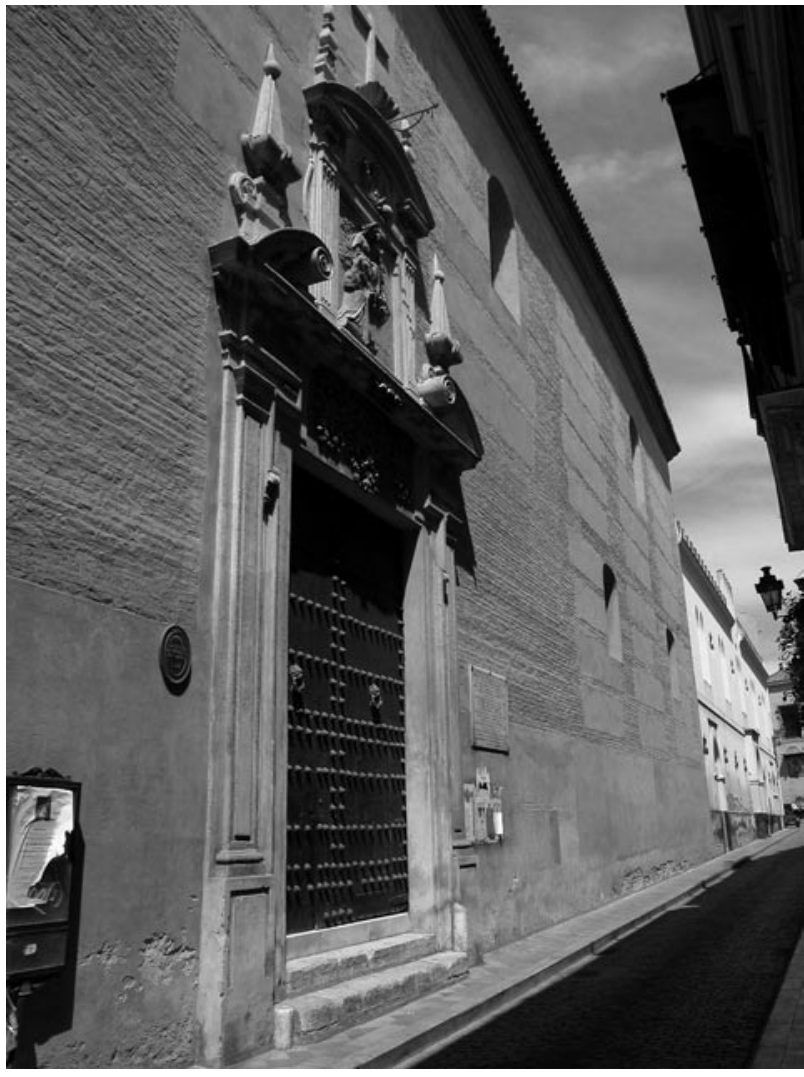

FIGURA 10. Portada del Convento Madre de Dios, sede de la Escuela Libre de Medicina y Cirugía de Sevilla, en la actualidad. desajuste que se observa en la distribución entre la actividad profesional asistencial y el puesto teórico docente ocupado es significativo, obedeciendo a la adjudicación que el propio Dr. Rubio y Galí realizaba de forma arbitraria. Las disciplinas clínicas quirúrgicas presentaron un mayor nivel de adecuación, siendo impartidas por cirujanos o especialistas de reconocido prestigio. Las clases daban comienzo a las 7 de la mañana y terminaban a las 4 de la tarde. El número de alumnos se muestra en la Tabla II.

Los exámenes se realizaban ante un tribunal conformado por dos profesores de la Escuela y un tercer médico ajeno a ella. En 1871 se licenciaron 2 alumnos, 4 alumnos en 1872, y 3 en 1874, sumando cifras semejantes los que consiguieron el grado de doctor.

Las crónicas refieren el buen desarrollo de la Escuela Libre de Medicina y Cirugía de Sevilla y, aunque fueron varios los establecimientos libres que se crearon, fue la Escuela de Medicina la que mayor protagonismo logró. Sin duda achacable a la estrecha relación con la vida social de una ciudad que siempre le reconoció su constante labor sanitaria y humanitaria, en beneficio de una población tan necesitada de su asistencia.

Pero en su recorrido histórico, la Escuela sevillana tuvo que adaptarse a las distintas circunstancias que la legislación de la época imponía, con

TABLA II. NÚMERO DE ALUMNOS.

\begin{tabular}{|c|c|c|c|c|c|}
\hline PERIODO & CURSO & MATRICULAS & N/P & APROBADOS & SUSPENSOS \\
\hline $1869 / 70$ & $1^{\circ}$ & 98 & 39 & 51 & 8 \\
\hline $1869 / 70$ & $2^{\circ}$ & 76 & 26 & 50 & 3 \\
\hline $1870 / 71$ & $1^{\circ}$ & 158 & 35 & 93 & 30 \\
\hline $1870 / 71$ & $2^{\circ}$ & 112 & 32 & 67 & 13 \\
\hline $1871 / 72$ & $1^{\circ}$ & 171 & 63 & 79 & 29 \\
\hline $1871 / 72$ & $2^{\circ}$ & 142 & 72 & 54 & 16 \\
\hline $1872 / 73$ & $1^{\circ}$ & 154 & 35 & 93 & 26 \\
\hline $1872 / 73$ & $2^{\circ}$ & 91 & 20 & 61 & 10 \\
\hline $1873 / 74$ & $1^{\circ}$ & 151 & 62 & 64 & 25 \\
\hline $1873 / 74$ & $2^{\circ}$ & 88 & 11 & 69 & 8 \\
\hline
\end{tabular}


tal de lograr su continuidad. Muy a su pesar, y en pos de su permanencia, tuvo que hacer grandes esfuerzos que en no pocas ocasiones se tradujeron en supresión de disciplinas, asimilación a las directrices de la enseñanza de carácter oficial y disminución de costos. Así, en 1875 la Escuela Libre de Medicina y Cirugía pasó a denominarse "Escuela Provincial de Medicina de Sevilla".

El número de alumnos comenzó a bajar a partir de 1882, pasando de los 1.305 alumnos matriculados durante el curso 1881-1882 a los 931 del curso 1882-1883, culminando la decadencia en el curso 1894 a 1895 con tan solo 427 alumnos matriculados. En 1902 la Escuela Provincial de Medicina se transformó en la oficial Facultad Provincial de Medicina y Cirugía, como única salida encontrada para evitar su desaparición total.

\section{La Escuela Libre de Medicina y Cirugía de Sevilla y su aportación a la Urología.}

En primer lugar conviene aclarar que el término Urología como tal Especialidad Médica que nos ocupa, fue acuñado con posterioridad a la fecha que estudiamos (1896); no obstante y hecha esta salvedad, lo emplearemos en nuestro trabajo.

A nivel general, la Urología en la Escuela se benefició de numerosas aportaciones, la principal de las cuales fue su incorporación a la Cirugía moderna y a las nuevas corrientes científicos-médicas llegadas del exterior que, al igual que otras ciencias, fueron difundidas y auspiciadas por su mentor, el Doctor Federico Rubio y Galí.

Gracias a la Escuela Libre de Medicina y Cirugía de Sevilla se inició la enseñanza reglada de la Urología como disciplina. El plan de estudios de la Escuela contempló estudiar tres materias no obligatorias en la enseñanza oficial, entre ellas "Afectos Venéreos y su Clínica". Posteriormente las clínicas de "Enfermedades de las Vías Urinarias" y la de "Enfermedades Sifilíiticas" supieron abrirse poco a poco paso como disciplina, pasando en los años ochenta de ser contempladas como meros "cursos especiales" a ocupar tan sólo ocho años después la planta alta de la Policlínica, sentándose las bases de la Urología como Especialidad Médica. Entre los profesores encargados de impartir ambas disciplinas figuran: Jerónimo Sánchez Flores en el primer curso de la Escuela, ocupándose de "Afectos Venéreos". De 1870 a 1871 fue Gabriel Tejada encargado de la docencia de "Clínica Quirúrgica y Afectos Venéreos". En 1873 la impartió el Dr. Enrique Romero Pedreño y a partir de 1888 Manuel Moreno Parra fue profesor de "En- fermedades Venéreas y Sifilíticas" actuando Miguel Martínez como ayudante mientras Jacinto Bracho Quijano y Romero de la Vega impartieron "Enfermedades de las Vías Urinarias".

Por otra parte, la creación de la Policlínica potenció la práctica médica con el consiguiente estudio y seguimiento hospitalario de las clínicas relacionadas con las enfermedades del tracto urinario e infecciones venéreas. La recogida de información del paciente era vertida en una hoja clínica (una por cada paciente y en cada sesión), favoreciendo así el intercambio de experiencia clínica, y con ella la investigación y el estudio Urológico.

Las consultas en ambulatorio, y en particular la Consulta de Vías Urinarias, posibilitaron a los aspirantes a médicos el conocimiento y seguimiento de enfermedades Urológicas que difícilmente eran tratadas en régimen hospitalario. Además, los Urólogos pudieron desarrollar con el trato día a día, un contacto directo médico-paciente que les permitió no únicamente tratar su enfermedad, sino conocer la realidad cotidiana de sus enfermos pertenecientes a las clases sociales desfavorecidas, que soportaban unas duras condiciones de vida que complicaban su proceso patológico. Aquellos, tuvieron oportunidad de iniciar una Medicina Preventiva difundiendo prácticas profilácticas y de higiene personal que posibilitaran la prevención de las enfermedades venéreas y fomentaran la salud en general. Todo ello en consonancia con la introducción en España y en este siglo de los principios higienistas.

En relación a la resonancia de la práctica Urológica de la Escuela y las etapas posteriores de Don Federico Rubio y Galí en lo que a publicaciones científicas respecta, son numerosas las referencias bibliográficas al tema, de las que hemos destacado las siguientes: en 1873 el Libro de Actas de las Sesiones Literarias de la Academia Nacional de Medicina recoge una intervención del Doctor Rubio y Galí relacionado con un caso Urológico. En el Siglo Médico, en 1876 escribió sobre la necesidad de la circuncisión, tema que por otra parte desarrolló en una obra dedicada sobre el tema "De la Circuncisión", 1879, en la que defendió la necesidad de su práctica generalizada en varones por considerar el prepucio una seria amenaza para la salud tanto individual como colectiva. En 1890 en los Anales de la Real Academia Nacional de Medicina se recogen sus observaciones sobre "Pionefrosis con extirpación del riñón derecho. Curación", que también fue publicado en El Siglo Médico en el mismo año. Por último, en el "Tratado de Cirugía, Clínica y Operatoria" (1899-1903), en el volumen no 9, el Doctor Rubio y Galí comentó los artículos de Leguen sobre "Enferme- 


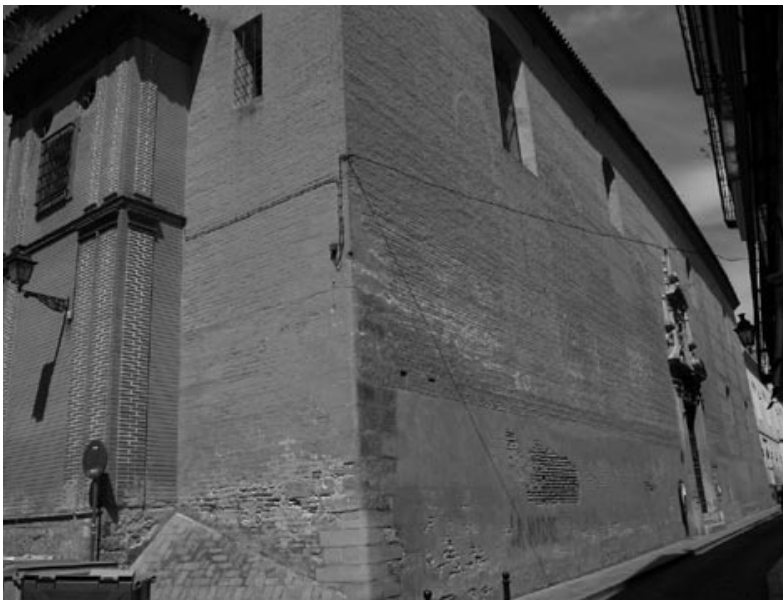

FIGURA 1 1. Piso alto del Convento Madre de Dios, dependencias ocupadas por la Policlínica Urológica, en la actualidad.

dades de la Vejiga" e "Infecciones Urinarias", y el artículo de Albarrán "Enfermedades de la Uretra".

Esta destacable producción académica provenía de un productivo ejercicio clínico de eminente enfoque práctico, con la instauración de sesiones clínicas regladas en las que se discutían aspectos y particularidades de las distintas patologías estudiadas. Fruto de ello fueron las consideraciones que se efectuaban sobre aspectos concretos, como ilustran los siguientes ejemplos:

- Sobre el caso de la Nefrostomía: maniobra renal tanto exploradora como terapéutica de orden percutáneo, según Maganto, realizada por primera vez en España por el Doctor Rubio y Galí.

- En una cuestión que hoy en día nos parece tan clara, Don Federico Rubio estableció la necesidad de asegurar una correcta función renal contralateral antes de acometer cualquier cirugía sobre un riñón enfermo, así como extremar las precauciones en casos de riñón único.

- El Doctor Rubio y Galí destaca la necesidad de extremar la pulcritud en la cirugía de la Uretra por el peligro de la hemorragia postoperatoria, en especial la subsecuente a la Uretrotomía interna y las dificultades de la resección en los casos de estenosis.

- Como observación pionera, advirtió de la importancia en el manejo de todos los pacientes, aunque de forma especial en los que padecen enfermedades de los órganos sexuales, de los aspectos psicológicos de éstos, insistiendo en el estudio de esta faceta.

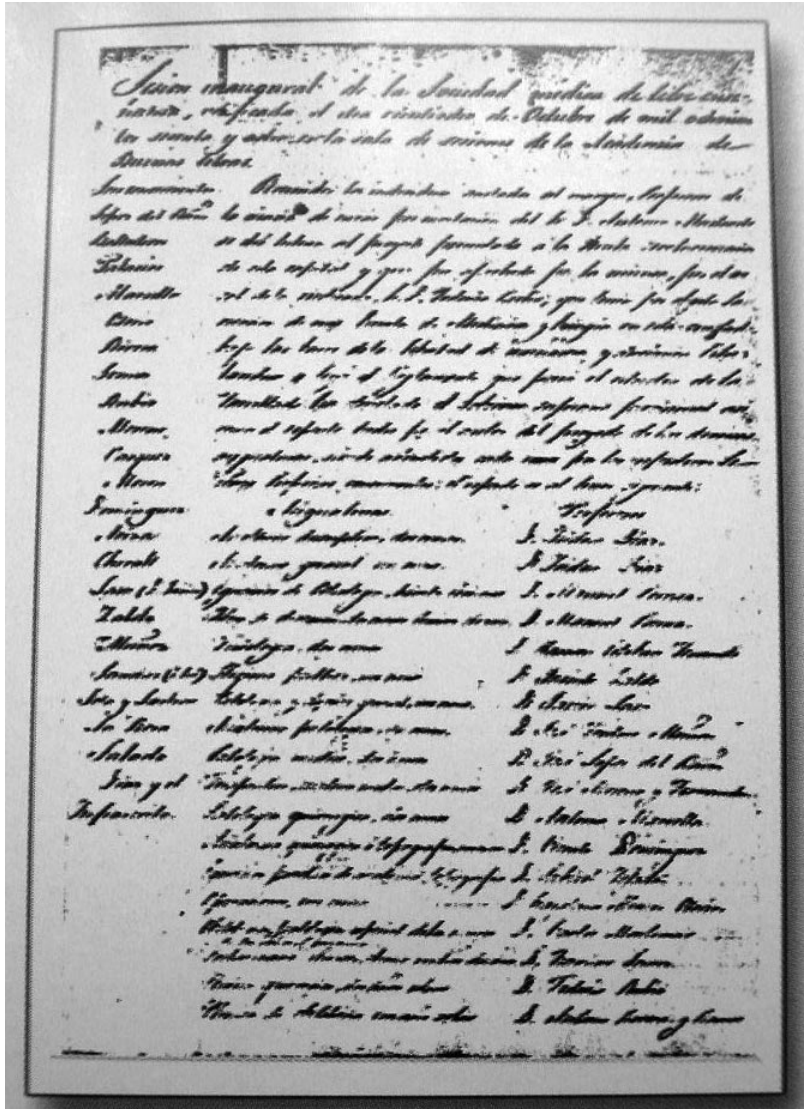

FIGURA 12. Acta de la primera reunión científica de la Escuela Libre de Medicina y Cirugía de Sevilla. Año 1868.

- Llevado de su tendencia a las maniobras mínimamente invasivas, siempre se mostró favorable a la exploración endoscópica, ya fuere mediante el espéculo vaginal, el rectoscopio o incluso el laringoscopio.

- Como comentario de técnica operatoria de estas sesiones, se mencionan dos intervenciones relacionadas con la Penectomía y con la Emasculación Escrotal. En el caso de la primera, el principal inconveniente aducido fue la hemorragia así como la flojedad de la piel, la retractilidad de los cuerpos cavernosos y la estenosis uretral; esto último constituía un importante problema tanto por su frecuencia como por lo engorroso que resultaba tener que usar de forma permanente una cánula de plata que no siempre la evitaba. Como resultado de su experiencia, introdujo unas modificaciones, que de forma resumida se pueden describir como un vendaje especial que impedía la retracción de la piel y la flaccidez del miembro, así como la sección con tijeras siguiendo unas direc- 
ALBUM OE LA . REVISTA MEDICA DE SEVILLA.

EXMO. SR. DR. D. FEDERICO RUBIO Y GALI

(APUNTES PARA UNA SEMOLANZA)

$$
=
$$

Reexo. Press ya que te fijastes biea en la bíblica figera del folograbada

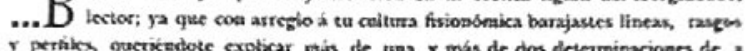

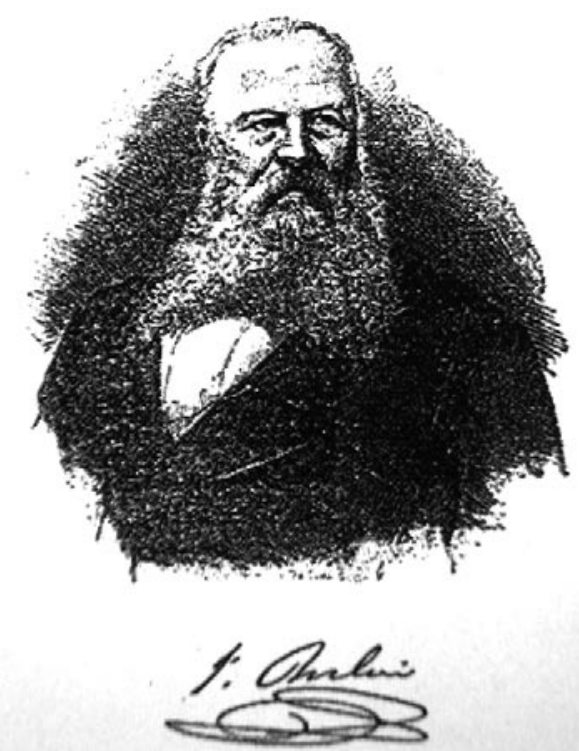

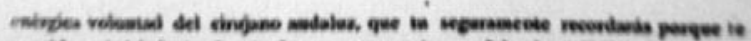

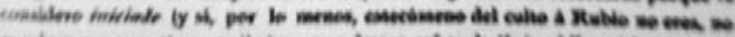

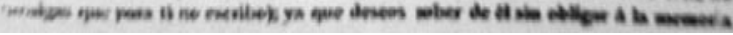

FIGURA 13. Revista Médica de Sevilla. Ejemplar de semblanza y homenaje al Dr. Federico Rubio y Galí.

trices, con un especial diseño de la incisión y de la forma de suturarla. La emasculación total, según sus manifestaciones, la realizó por necesidad de un enfermo que padecía un epitelioma del miembro.

\section{CONCLUSIONES}

Las autoridades locales de la Sevilla del XIX, se enfrentaron al reto de dar solución al problema de la salud pública de sus conciudadanos. Desde un principio reconocieron su impotencia, si bien trataron por todos los medios de favorecer cuantas iniciativas sanitarias se produjeran en la ciudad. Un claro ejemplo lo encontramos en el apoyo otorgado a la Escuela Libre de Medicina y Cirugía.

La Medicina se benefició de las aportaciones científicas producidas a lo largo del siglo y del ambiente liberal que le permitió desarrollar la praxis. Hubo una percepción real sobre la relación existente

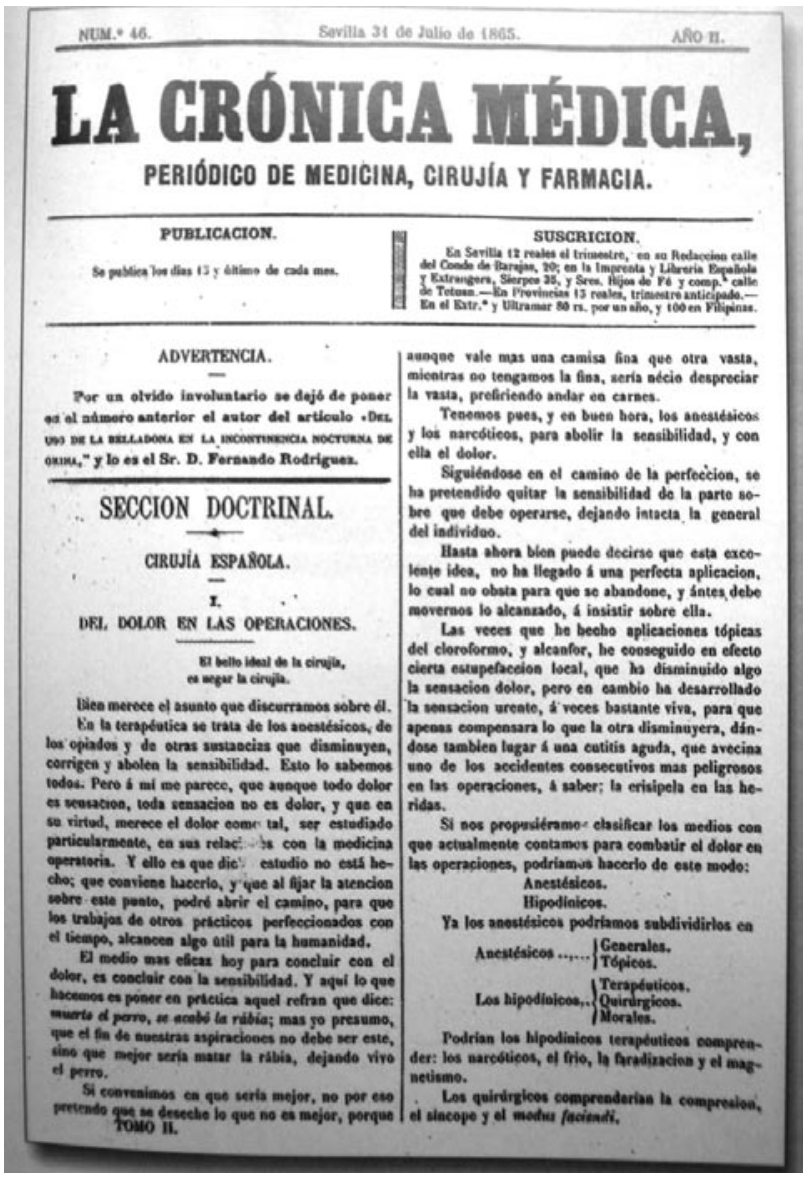

FIGURA 14. Revista La Crónica Médica. Ejemplar en el que aparece el artículo "Del Dolor en las Operaciones", del Dr. Federico Rubio y Gali. Año 1865.

entre los niveles sociales menos favorecidos de la población y la persistencia en estos grupos de determinadas enfermedades en las que el factor higiene era fundamental.

La Escuela Libre de Medicina y Cirugía de Sevilla contribuyó, dentro de sus posibilidades y recursos, a mejorar la atención sanitaria en la ciudad hispalense.

El legado del Doctor Don Federico Rubio y Galí a la Historia de la Medicina en general y a la Urología en especial es indudable. Gracias a sus esfuerzos, la Urología desde unos tímidos inicios fue adquiriendo protagonismo y desarrollo en la práctica de la Medicina. De hecho, en 1873 Federico Rubio presentó en Sevilla un expediente para que fuera autorizada la creación de una Cátedra de Enfermedades de las Vías Urinarias, no cesando de propugnar a lo largo de toda su vida la importancia de la patología Urológica. 


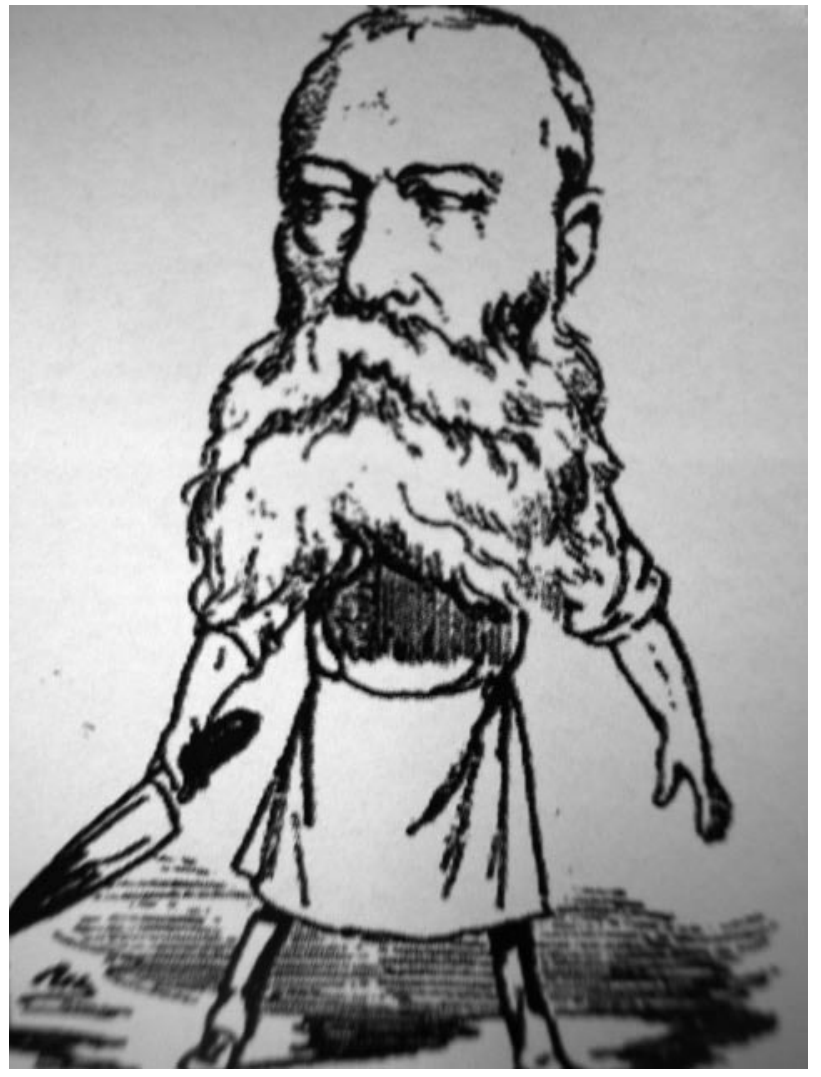

FIGURA 15. Caricatura del Dr. Federico Rubio y Galí. El afán quirúrgico del andaluz fue motivo de mofa y escarnio a mano de sus detractores, que lo presentaban más cercano a un matarife que a un médico.

Como en otros campos, el Doctor Federico Rubio favoreció tanto la aplicación en Urología de la Cirugía moderna como la introducción de las nuevas corrientes científicas, ya que el Doctor Rubio y Galí, más que un innovador, fue un gran reformador y difusor en España de los conocimientos y avances médicos que tuvo ocasión de conocer en países avanzados científicamente como Reino Unido, Francia, o Estados Unidos.
Debemos matizar que pese a la consideración del Doctor Rubio y Galí como un gran cirujano, su práctica no estuvo exenta de fracasos: él mismo se quejaba de no haber podido conseguir dominar la utilización de la Anestesia en sus pacientes; o bien el hecho de que en alguna de sus conocidas y celebradas operaciones los pacientes sobrevivieran sólo unos cuantos días a la intervención practicada. No obstante, Don Federico Rubio siempre optó por llevar a cabo las operaciones quirúrgicas menos invasivas y que menor peligro entrañaran al paciente para evitar, precisamente, su muerte como causa directa de la intervención.

Por todo lo expuesto, creemos más que justificado considerar al Doctor Don Federico Rubio y Galí como el médico que más ha contribuido a la creación de la especialidad de la Urología, recibiendo merecidamente el título de "Príncipe de la Cirugía".

\section{BIBLIOGRAFÍA y LECTURAS RECOMENDADAS (*lectura de interés $y^{* *}$ lectura fundamental)}

1. "Medicina y Sociedad en la España de la segunda mitad del Siglo XIX: Una aproximación a la obra de Federico Rubio y Galí (1827-1902)”. Editor: Juan Luis Carillo Martos. El Puerto de Santa Maria, 2003.

2. "Catalogo de la Exposición. El Dr. Federico Rubio y la renovación de la medicina española (1827-1902)". Ayuntamiento de El Puerto de Santa María, 2002.

3. "La enseñanza de la medicina en la Universidad Española". Coordina: José Danón. Fundación Uriach, Colección Histórica de Ciencias de la Salud, 1998.

4. CARRILO, J.L.: "Federico Rubio y Galí (18271902): Estudio documental y bibliográfico". El Puerto de Santa María, 2002. 